\title{
Interactive comment on "Assessing the impacts of reservoirs on the downstream flood frequency by coupling the effect of the scheduling-related multivariate rainfall into an indicator of reservoir effects" by Bin Xiong et al.
}

Anonymous Referee \#1

Received and published: 16 April 2019

This study describes a modeling framework to account for the role of reservoirs in flood frequency analysis. While I think that the topic is generally of interest to the readership of this journal, I have a number of comments that should be addressed before considering it for publication.

- The manuscript needs to be proofread more carefully as there are several typos and unclear sentences. I will try point out some of these issues in the comments below, but this is not a complete list. 
- Line 26: what "previous study"?

- Lines 46-49: which of the two references is the quote from?

- Line 49: "nature extreme flow" is unclear.

- Line 46: "this method makes it suitable"

- Line 77: "the first approach". Also, please add a reference to support the statement.

- Lines 95-96: unclear why you can't get the uncertainties in the estimates. Please clarify.

- Line 98: "all their cases"

- Line 104: "for the expression of the distribution"

- Line 106: "in the expression"

- Given that you use a GEV but leave the shape parameter constant (and this is fine), please add more 2-parameter distributions (e.g., lognormal, gamma, Weibull, Gumbel) which have only two parameters that you can make vary as a function of your covariates.

- Line 132: "To analyze"

- Line 139: "The Eq. (1)"

- If I get this right, you are assuming that the sediment trapping capability of the reservoir is negligible. However, over time the amount of storage decreases. To account for the role of sediment in reducing the reservoir capacity over time, I highly recommend the use of the Brune curve to account for it. If not Brune curve, please account for it in some fashion.

Printer-friendly version

- Line 157: "the greater the MRI impact"

Discussion paper

- Line 158: what does "inflexible" mean in this context? 
- Line 161: "where"

- In terms of predictors, the spatial distribution of rainfall is not really captured. I can think of situations in which the same basin-averaged rainfall will have very different effects if most of the rainfall occurs far or close to the outlet. How is this addressed here?

- Line 185: "marginals"

- Line 204: "extensively concerned" is unclear.

- Line 208: what does "obeys nonstationary distribution" mean?

- What about model selection based on the SBC index? Would you get a more parsimonious model?

- Line 254: I don't think this statement is correct, given that you would be able to say whether a more complex model should be selected over a more complex one, not if the fit is good or bad.

- Line 266: “, and was completed”

- Line 281: what is the definition of "timing"?

- Line 303: what does "special" mean?

- Line 314: "was calculated"

- In fitting the copulas, the marginals were treating as stationary. Is this really the case? Please test for the presence of nonstationarities in the marginals of the predictors. If nonstationary, please account for it.

- The role of the Mann-Kendall and Pettitt tests is unclear to me. First of all, the results are discussed at a very basic and superficial level. Also, if the response variable tends to change with time but because the predictors you have selected change over time as well, then whether $Y$ is stationary or not is not very important; however, whether 
the relationship between predictors and predictand doesn't change over time becomes more relevant. Please fix this part.

- Lines 362-364: Please apply a correction to account for the fact you are performing multiple hypothesis testing

- Line 374: "explains"

- Line 391: "for every certain multivariate MRI" is unclear.

- Line 402: "It is of interest"

- Line 404: "the remaining capacity of the reservoir"

- Line 409: "due to correspond to" is unclear

- Line 423: "related to the construction"

- Line 427: "is weak"; "The comparison"

- Line 428: "indicates"

- Line 429: "in most cases"

- Line 435: "100-year"

- Line 649: "thick blue" what?

- Line 651: "The right panels are"

Interactive comment on Hydrol. Earth Syst. Sci. Discuss., https://doi.org/10.5194/hess-201942, 2019. 